\title{
FROM CONTEXT TO CODE: SHARED INFORMATION CONSTRAINS THE EVOLUTION OF OPTIMAL CODES
}

\author{
JAMES WINTERS $^{* 1}$ and OLIVIER MORIN ${ }^{1}$ \\ *Corresponding Author: winters@shh.mpg.de \\ ${ }^{1}$ The Minds and Traditions Research Group, Max Planck for SHH, Jena, Germany
}

We communicate by means of coded information, contained in spoken words, written signs, or gestures. A code is a set of reliable associations between signals and meanings used to facilitate information transfer (Scott-Phillips, 2014). The amount of information that can be extracted from a code depends on its stability (the regularity of a signal-meaning mapping), and on whether the set of signalmeaning associations form an optimal configuration. A code is optimal when it reaches a tradeoff between two constraints. First, it contains the relevant information that the Recipient is supposed to extract from it (informativeness). Second, it presents this information in a compressible format, using mappings with a minimal description length (compressibility). Optimal codes are therefore the most compressible set of signal-meaning mappings capable of identifying the intended meaning in context (Kirby et al., 2015).

Optimal codes help facilitate successful communication, but are not indispensable. Efficient communication can happen even with an unstable and weak code - because it makes use of information that is contextual as opposed to encoded (Piantadosi et al., 2012). Context, in this sense, is information external to a code which is relevant for reducing uncertainty about a Messenger's intended meaning. This contextual information can be retrieved from the common environment that communicators share; it can be stored in memory; or it can be inferred from a combination of these sources (Clark \& Brennan, 1991). Only when the context is sufficiently rich to allow for some information transfer to take place, do optimal codes emerge to fill and enrich expressive gaps in communication (Bybee, 2010).

We test the hypothesis that the informational distance between a Messenger and a Recipient (i.e., the amount of shared information) acts as a 
strong constraint on solutions to this context-coding problem. Affecting the amount of shared information is whether the mode of information transfer is synchronous or asynchronous and whether the function of information transfer is monadic or dyadic. Synchrony, which we define as information transfer within the same timeframe, leverages the presence of shared contextual information, as well as the ability to provide feedback about informativeness, to increase the amount of shared information. Asynchrony, on the other hand, is where information transfer takes place across timeframes, reducing access to shared contextual information and removing availability of immediate feedback. Monadic information transfer, where information is transferred within an individual, corresponds to an increase in shared information: individuals must only coordinate with themselves to facilitate successful information transfer. This is in contrast to when information transfer is dyadic: Messenger and Recipient are different individuals, making coordination a more substantial problem as there is less shared information.

Using a referential game set up, where participants play as Messengers and Recipients, we experimentally manipulate both the mode (synchronous or asynchronous) and function of information transfer (monad or dyad) to generate four conditions: Recall (synchronous + monad), Mnemonic (asynchronous + monad), Dialogue (synchronous + dyad), and Correspondence (asynchronous + dyad). Only in Dialogue, where Messengers and Recipients are two different persons interacting within the same timeframe, do we consistently observe the emergence of stable and optimal codes. In the Mnemonic condition, where Messenger and Recipient are the same person at different points in time, participants produce informative codes that are comparatively less stable and compressible than those in Dialogue. By contrast, in the Recall condition, where Messenger and Recipient are the same person at the same point in time, and the Correspondence condition, where Messenger and Recipient are two different persons communicating across timeframes, stable and optimal codes fail to emerge.

These results demonstrate that solutions to the context-coding problem are contingent on the amount of shared information. If interlocutors share access to the same perceptual context, and have recourse to immediate feedback on their performance, then Messengers can leverage this shared information to rapidly coordinate with Recipients in constructing an optimal code. This builds on the idea that human communication is adapted to synchronous interactions between individuals (Levinson, 2006); codes can readily leave out information already provided by the context, and miscommunications can be repaired on the fly. 
When the distance between interlocutors increases, as is the case in the Correspondence condition, Messengers are unable to estimate, and therefore exploit, the information they share with the Recipients, resulting in unstable and nonoptimal codes.

\section{References}

Bybee, J. (2010). Language, usage and cognition. Cambridge University Press.

Clark, H. H., \& Brennan, S. E. (1991). Grounding in communication. In L. Resnick, L. B., M. John, S. Teasley, \& D. (Eds.), Perspectives on socially shared cognition (pp. 13-1991). American Psychological Association.

Kirby, S., Tamariz, M., Cornish, H., \& Smith, K. (2015). Compression and communication in the cultural evolution of linguistic structure. Cognition, 141, 87-102.

Levinson, S. C. (2006). On the human interaction engine. In N. J. E. . S. C. Levinson (Ed.), Roots of human sociality: Culture, cognition and interaction (p. 39-69). Oxford: Berg.

Piantadosi, S. T., Tily, H., \& Gibson, E. (2012). The communicative function of ambiguity in language. Cognition, 122(3), 280-291.

Scott-Phillips, T. C. (2014). Speaking Our Minds. Macmillan Education UK. 